\title{
Das Projekt "Reformationsatlas"
}

\section{Karten zur Reformation in Mitteldeutschland}

\author{
Markus Hein
}

Das diesjährige Reformationsjubiläum, das seit Jahren die öffentliche Wahrnehmung von Kirche prägt - zumindest in Sachsen und Mitteldeutschland -, hat eine Unzahl an Veröffentlichungen hervorgebracht und wird dies auch in diesem Jahr noch tun. Sei es, dass diese sich mit einzelnen Ereignissen der Reformationsgeschichte wie dem das Jubiläum auslösenden Anschlag der 95 Thesen Martin Luthers gegen den Missbrauch des Ablasses am 31. Oktober 1517 beschäftigen oder mit Personen - insbesondere Luther selbst - bzw. Personengruppen (Fürsten, Mitreformatoren) auseinandersetzen, sie alle eint, dass es ein mehr oder weniger klassisches Herangehen ist. Das Ergebnis sind Aufsatzbände oder Monographien, die auf mal mehr, mal weniger Seiten Text den Leser oder die Leserin in die Ereignisse mitnehmen, oft auch von Bildern begleitet. Daneben gab und gibt es in den letzten Jahren viele Ausstellungen, die das Reformationsjubiläum zum Anlass nahmen und nehmen, und die nicht nur mit Texten, sondern auch mit Gemälden und Objekten die tiefgreifenden Änderungen dieser Epochenumbruchszeit nahe zu bringen versuchen. Das ist gut so, das ist üblich und das war erwartet und bringt der Erforschung der Frühen Neuzeit einen gewaltigen Schub, weit über das engere Geschehen der deutschen Reformation hinaus.

Im Folgenden nun soll ein bisher kaum beachtetes Genre in das Blickfeld gerückt werden, das auf ganz andere Art versucht, historisches Geschehen und historische Umbrüche sichtbar und verstehbar zu machen: der Atlas, der versucht, das Geschehen im Wesentlichen kartographisch darzustellen.

Am Institut für Kirchengeschichte in Leipzig entsteht am Lehrstuhl von Prof. Dr. Armin Kohnle (Spätmittelalter, Reformation und Territorialkirchengeschichte) im Moment ein solcher Atlas, der die Geschichte der Reformation in Mitteldeutschland in den Mittelpunkt rückt. Auf ca. 150 Doppelseiten soll dargelegt werden, wie die Reformation in den einzelnen Regionen und auf den verschiedenen Gebieten des kirchlichen und gesellschaftlichen Lebens vor sich ging. „Atlas“ ist hierbei ganz im klassi- schen Sinne gemeint. Überwiegend sind die Seiten von Karten dominiert. Karten bieten einen anderen Zugang, auch und gerade zu Geschichte. Mit ihrer Hilfe lassen sich nicht nur andere und mehr Sinne ansprechen, mit ihnen lassen sich auch verschiedene Zusammenhänge und Ereignisse anders und vielleicht plausibler verdeutlichen. Dabei liefern Karten in der Regel komplexere Informationen, als es ein Text kann, und haben eine faszinierende Anziehungskraft. U. a. können Entfernungen und Bewegungen sichtbar gemacht werden, Größenverhältnisse und Verbindungen von Personen untereinander oder zu Orten. Auf einen Blick ist in der Regel mehr zu erfassen als auf einer Illustration oder in einem Text.

Ziel ist es, einen Atlas zu erstellen, der auf den neuesten Forschungen zur Reformation beruht. Zielgruppe sind dabei nicht nur Forschungseinrichtungen oder Lehranstalten wie Universitäten und Schulen, sondern vor allem auch an Religion und Geschichte interessierte Laien. Das Reformationsjubiläum und die Lutherdekade haben die Reformation und ihre Geschehnisse wieder einem deutlich breiteren Kreis näher gebracht, für den hier ein über das Übliche hinaus gehender Zugang gegeben werden soll.

Begonnen wurde mit dem Projekt - nach einem Vorlauf - Anfang 2013. Die Ostdeutsche Sparkassenstiftung ist wichtigster Fördermittelgeber des Projektes, das auch von der Universität Leipzig nicht unerheblich unterstützt wird. Die Umsetzung der Karten erfolgt an der Hochschule für Technik und Wirtschaft (HTW) Dresden, wo mit Prof. Dr. Uwe Ulrich Jäschke ein Kenner der historischen Kartographie zur Verfügung steht. Dort ist auch der „Atlas zur Geschichte und Landeskunde Sachsens“ entstanden, so dass man um die Schwierigkeiten von historischen Karten weiß, wenn auch eine Nutzung in der Gegenwart angestrebt wird.

Neben dem Buchatlanten entsteht natürlich auch eine Onlinevariante, die die im Buch niedergelegten Ergebnisse für das Netz nutzbar macht, wobei es nicht einfach um eine Wiedergabe der Buchseiten geht. Kombinierte Suchfunktionen und Verknüpfungen mit anderen 
Websites sollen den Atlas und vor allem den Inhalt desselben auch weniger buchaffinen Menschen näher bringen. Darüber hinaus bietet dies auch vielfältige Möglichkeiten des Weiterarbeitens und -verbreitens - sowohl für Schule, Touristik und Forschung. Verantwortlich für diese Umsetzung ist das geoinformatische Institut der HTW Dresden unter der Leitung von Frau Professor Dr. Martina Müller.

Die grafische Gestaltung der Seiten hat das Büro Formenorm (Friederike Arndt) in Leipzig übernommen, das aus den Karten, Texten, Bildern und Ideen in enger Absprache die Doppelseiten erstellt. Erscheinen wird der Atlas beim in Dößel bei Halle ansässigen Verlag Janos Stekovics.

Die Doppelseiten bieten immer eine attraktive Kombination aus Texten, Bildern, Grafiken und Karten, wobei mindestens 50 Prozent kartographisches Material angestrebt ist. Im Idealfall sollen die Texte erläutern und Zusammenhänge herstellen, der Schwerpunkt aber auf der Visualisierung reformationsgeschichtlicher Prozesse liegen.

Nicht immer ist dies möglich, soll doch der Atlas nicht nur Ausschnitte, sondern das ganze Geschehen der Reformation in Mitteldeutschland zum Thema haben. Neben den Ereignissen der Reformation des 16. Jahrhunderts selbst werden auch die Wirkungen der Reformation für Kirche und Gesellschaft, für Kultur, Politik und Wirtschaft in den Blick genommen werden - ein Anspruch, der wesentlich schwieriger zu bearbeiten ist, als er klingt.

Konzipiert ist der Reformationsatlas auf Mitteldeutschland hin. Darunter wird das historische (wettinische) Sachsen verstanden mitsamt seine kleineren Nachbarn. Das entspricht nicht nur den politischen und kirchlichen Verhältnissen der Reformationszeit, sondern es ist auch ohne Mühe mit den heutigen Bundesländern Sachsen, Sachsen-Anhalt und Thüringen in Deckung zu bringen, die auch im Jahr des Reformationsjubiläums die bedeutendsten Orte stellen. Thüringen ist der Raum, der vor allem mit der Biographie des ,jungen Luther“ verbunden ist (Eisenach und Erfurt), wo die Wartburg als der Erinnerungsort steht, der bis heute untrennbar mit der Bibelübersetzung verbunden wird. In Sachsen-Anhalt, das für „Durchbruch und Entfaltung“ der Reformation steht, finden wir einerseits die Luthers Leben und Familie umspannenden Orte Mansfeld und Eisleben, aber auch das seiner Reformation den Namen gebende Wittenberg, wo sich die „Wittenberger Reformation“, verbunden mit der dortigen Universität und Reformatoren wie Karlstadt und Melanchthon, ihren Kernort schuf. Der Frei- staats Sachsen steht für die „reformatorischen Wirkungen“, vor allem auf den Feldern Schule und Bildung (Fürstenschulen), Musik und Kultur, aber auch Politik und Wirtschaft.

Schon diese Formulierungen zeigen, dass auch hier zunächst noch eher von der schriftlichen Form her gedacht und geplant wurde. Allerdings wurde sehr schnell klar, dass man da, wo man bei Texten durchaus unklare Stellen umschiffen oder auch Erwägungen formulieren kann, bei Karten kein Spielraum besteht. Hier ist dies nur sehr bedingt möglich, es kommt gerade auf Genauigkeit und Exaktheit an. Dies hat zur Folge, dass manche Dinge und Themen sich auch gar nicht mit Hilfe von Karten darstellen lassen. Bei anderen muss man sehr viel genauer nachfragen und nachforschen und oftmals helfen die üblichen Aufsätze und Ausarbeitungen nicht weiter.

In Leipzig sind für die Arbeiten und Aufgaben am Atlas neben einem Wissenschaftlichen Mitarbeiter studentische Hilfskräfte und eine wissenschaftliche Hilfskraft als Teilzeitkräfte angestellt, die bei der Recherche und Bereitstellung der Daten, Bilder und Texte mitarbeiten. Dabei werden von vornherein auch alle Orte mit ihren geographischen Daten erfasst, um einer späteren Nutzung im Online-Bereich vorzuarbeiten.

Der Atlas wird in zehn Abteilungen gegliedert sein, die möglichst dem chronologischen Verlauf folgen. Inhaltliche Überschneidungen lassen sich dabei nicht immer vermeiden: I Mitteldeutschland vor der Reformation, II Luthers Heimat und früher Werdegang, III Die Wittenberger Reformation bis 1525, IV Das ernestinische Sachsen im Zeichen von Visitation und Konfession, V Das albertinische Sachsen, VI Luther und die Wittenberger Reformatoren, VII Krise und äußere Sicherung der Reformation 1546 bis 1555, VIII Eine Welt im Wandel, IX Ausdifferenzierung der Reformation und Entstehung von Konfessionskirchen im 16. Jahrhundert, X Wirkungen. Der Atlas soll im September 2017 erscheinen.

Auf den folgenden Seiten sind zwei Doppelseiten wiedergegeben, die auf der ersten das Visitationsgeschehen in den Wettinischen Gebieten zwischen 1525 und 1540 nachvollziehen. Dabei sind - soweit bekannt - auch die jeweiligen Visitationsteams angegeben.

Die zweite Doppelseite verzeichnet das Geschehen um die Wurzener Fehde 1542. Besonders deutlich wird das aktive Intervenieren der Reformatoren von Wittenberg aus, das im Zusammenspiel mit dem Eingreifen Philipps von Hessen letztlich eine militärische Auseinandersetzung zwischen den Wettinern verhinderte.

\author{
Autor \\ Dr. Markus Hein \\ Universität Leipzig \\ Theologische Fakultät \\ Lehrstuhl für Spätmittelal- \\ ter, Reformation und terri- \\ toriale Kichengeschichte \\ Martin-Luther-Ring 3 \\ 04109 Leipzig
}




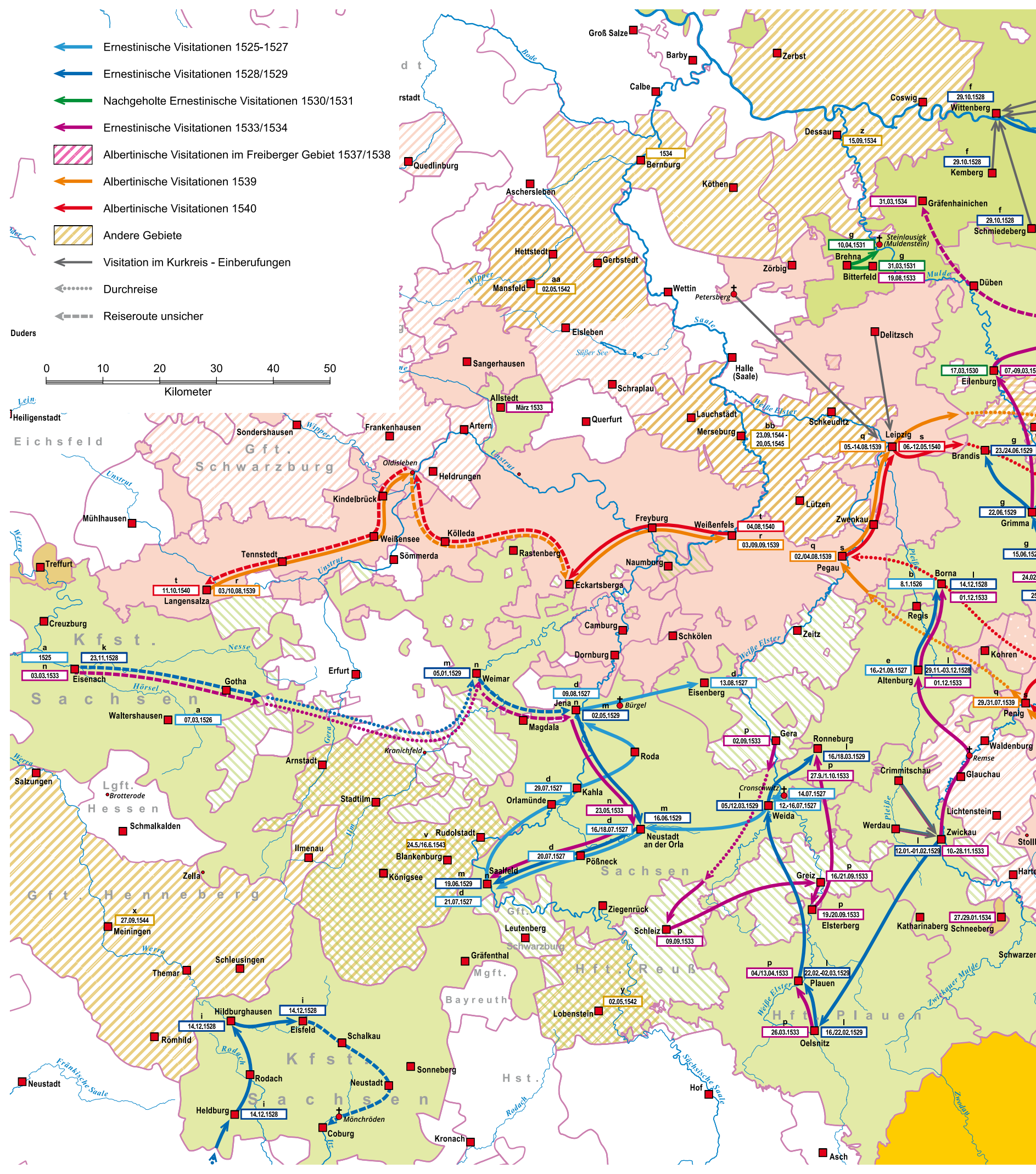




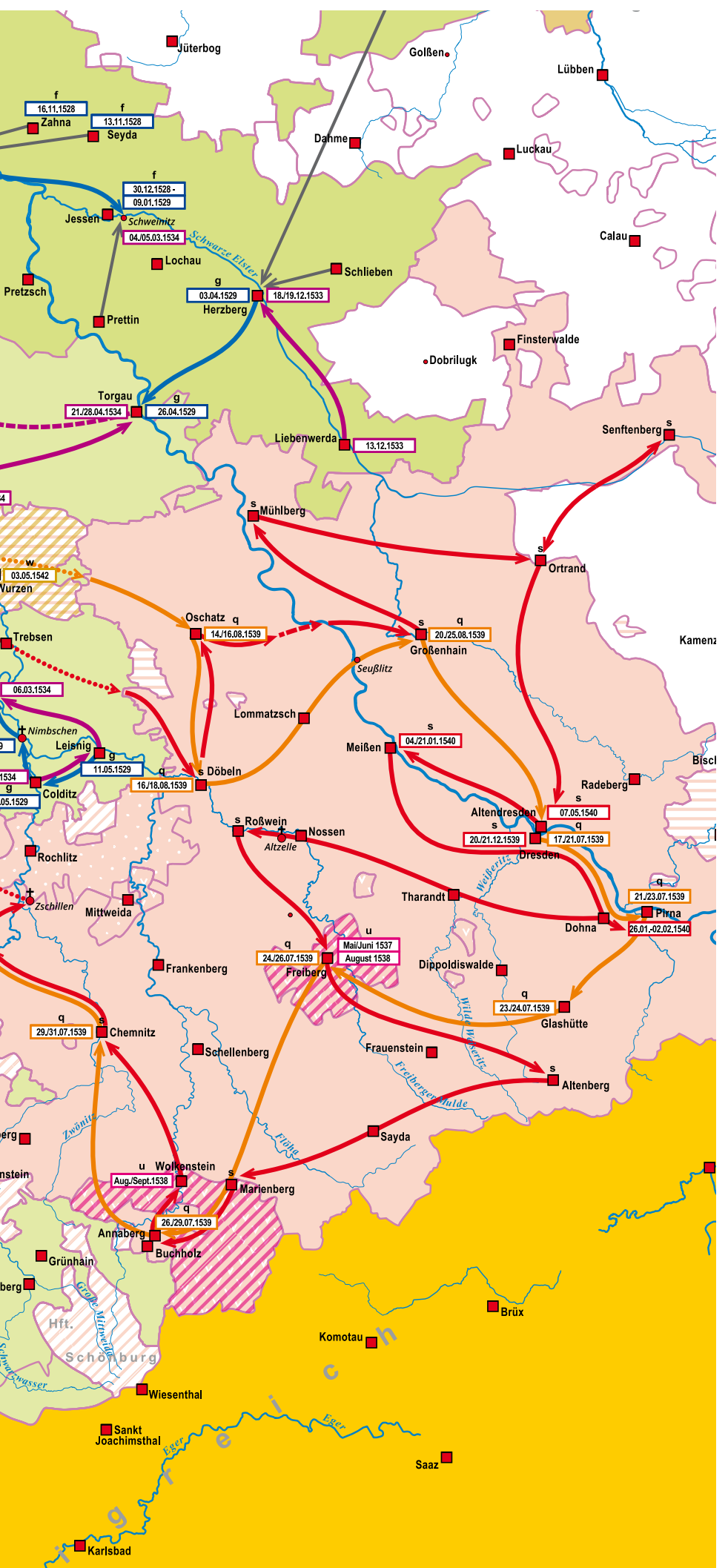

\section{Evangelische Visitationen}

Ernestinische Gebiete

a Jacob Strauß, Georg Witzel, Burkhard Hund-Um Eisenach, 1525

b Georg Spalatin, Michael von der Straßen - Amt Borna, 1526

c Friedrich Myconius, Johann Drach (Draconites) - Amt Tenneberg, 1526

d Philipp Melanchthon, Asmus von Haubitz, Hans von der Planitz, Hieronymus Schurff - Ostthüringen, 1527

e Philipp Melanchthon, Georg Spalatin, Asmus von Haubitz, Hans von der Planitz, - Altenburg, 1527

f Martin Luther, Justus Jonas, Hans Metzsch, Benedikt Pauli, Hans von Taubenheim - Kurkreis 1528/29

g Martin Luther, Wolfgang Fueß, Justus Jonas, Asmus von Haubitz, Bernhard von Hirschfeld, Sebastian von Kötteritz, Benedikt Pauli, Hans von Taubenheim, - „Unterer Meißnischer Kreis“ 1528/29

h Martin Luther, Johannes Bugenhagen, Justus Jonas, Nikolaus von Amsdorf (nur in Plötzky), Bernhard von Hirschfeld, Benedikt Pauli, Hans von Taubenheim - Kurkreis 1530

i Balthasar Düring, Nicolaus Kind, Hans von Sternberg, Paulus Bader Franken 1528

k Philipp Melanchthon, Justus Menius, Friedrich Myconius, Christoph von der Planitz - Westthüringen 1528/29

I Georg Spalatin, Anton Musa, Anarg von Wildenfels "Oberer Meißnischer Kreis“- 1528/29

m Georg Spalatin, Anton Musa, Ewald von Brandenstein, Christoph von der Planitz - Ostthüringen 1529

n Justus Menius, Friedrich Myconius, Georg von Wangenheim, Johann Cotta - Thüringen 1533

o Johannes Bugenhagen, Justus Jonas, Bernhard von Hirschfeld, Sebastian von Kötteritz, Caspar von Minkwitz - Kurkreis 1533/34

p Georg Spalatin, Johann Reimann, Christoph von der Planitz, Asmus Spiegel, Hans Metzsch, Michael Alber-Meißnischer Kreis und Reuß 1533/34

Albertinische Gebiete

q Justus Jonas, Georg Spalatin, Melchior von Kreutz, Rudolf von Rechenberg, Caspar von Schönberg - albertinisches Sachsen 1539

r Justus Menius, Johann Weber, Hartmann Goldacker, Friedrich von Hopfgarten, Volrad von Watzdorf - albertinisches Thüringen 1539

s Wolfgang Fueß, Caspar Zeuner, Hans von Kitzscher Rudolf von Rechenberg, Dietrich Preuß - albertinisches Sachsen 1540

t Wolfgang Fueß, Wolfgang Stein, Georg von Goldacker, Friedrich von Hayn, Christoph von Hopfgarten - albertinisches Thüringen 1540

u 1537: Georg Spalatin, Jacob Schenck, Melchior von Kreutz, Anton von Schönberg, Andreas Alnpeck

1538: Georg Spalatin, Leonhard Beyer, Caspar Freyberger, Melchior von Kreutz, Wolf Loß - „Freiberger Ländchen“ 1537/38

\section{Andere Gebiete}

v Johann Lang, Bonifatius Remp, Christoph Zwister, Lutz von Wüllersleben, Johann Zwister - Schwarzburg-Rudolstadt 1533

w Georg Spalatin, Johann Schreiner, Asmus Spiegel, Dietrich von Starschedel Amt und Stadt Wurzen 1542

x Johann Forster und einige gräfliche RäteHenneberg-Schleusingen (Meiningen) 1544

y Georg Rauth, Wolf von Gräfendorf - Reuß-Lobenstein 1543

z Nikolaus Hausmann, Gregor Peschel, zwei Laien - Anhalt-Dessau 1534 aa (Kaspar Güttel, Michael Cölius) - Mansfeld-Vorderort 1542

bb Georg von Anhalt, Anton Musa, Wolf von Bose, Ernst Brotauff, Jodocus Maier, Dietrich Riedel, Lorenz von Walthausen - Hochstift Merseburg 1545 


\section{Wurzener Fehde}

Das Wurzener Stiftsgebiet war Teil des Hochstifts Meißen. Seit der Leipziger Teilung 1485 stand es unter der gemeinsamen Schutzherrschaft der beiden sächsischen Linien. 1542 versuchte Kurfürst Johann Friedrich, seinen Einfluss auf Wurzen unter dem Vorwand verweigerter Türkensteuer, zu deren Zahlung sich Bischof Johann VIII. von Meißen ihm gegenüber nicht verpflichtet sah, auszuweiten. Am 22. März 1542 besetzten ernestinische Truppen die Stadt Wurzen. Sofort wurde der evangelische Gottesdienst eingeführt und die römische Messe verboten. Der Dom war künftig nur noch für Evangelische geöffnet, das Kirchengut wurde inventarisiert.

Der Bischof beschwerte sich beim albertinischen Herzog Moritz und bat ihn um Hilfe. Dieser mobilisierte seine Truppen, zog nach Oschatz und verlangte den Abzug der Ernestiner und ein gemeinsames Wahrnehmen der Schutzherrschaft. Johann Friedrich weigerte sich, Wurzen wieder freizugeben, bis alle seine Forderungen erfüllt waren, zog seinerseits in Wurzen eine Streitmacht zusammen und ließ die Stadt befestigen. Schließlich standen knapp 6.000 Soldaten bereit, und ein Krieg schien unausweichlich. Um diesen zu verhindern, schaltete sich Landgraf Philipp von Hessen zunächst mit Briefen, später mit persönlichen Vermittlungsversuchen zwischen den beiden sächsischen Fürsten ein. Auf Druck des Landgrafen, der von Luther und Melanchthon unterstützt wurde, konnte der Konflikt am 10. April durch einen Vertrag beigelegt werden. Dieser wurde in Oschatz durch Herzog Moritz, in Wurzen durch Landgraf Philipp und in Grimma durch Kurfürst Johann Friedrich verkündet und kam den Forderungen des Ernestiners weit entgegen. So wurden die reformatorischen Maßnahmen nicht rückgängig gemacht und eine Visitation angekündigt. Neben der Vermittlung des Landgrafen waren es vor allem die zum Frieden mahnenden Worte der Wittenberger Reformatoren, die dazu beitrugen, den Konflikt zu entschärfen.

\section{Schriftverkehr zur} Wurzener Fehde

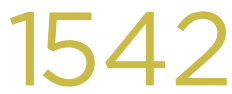

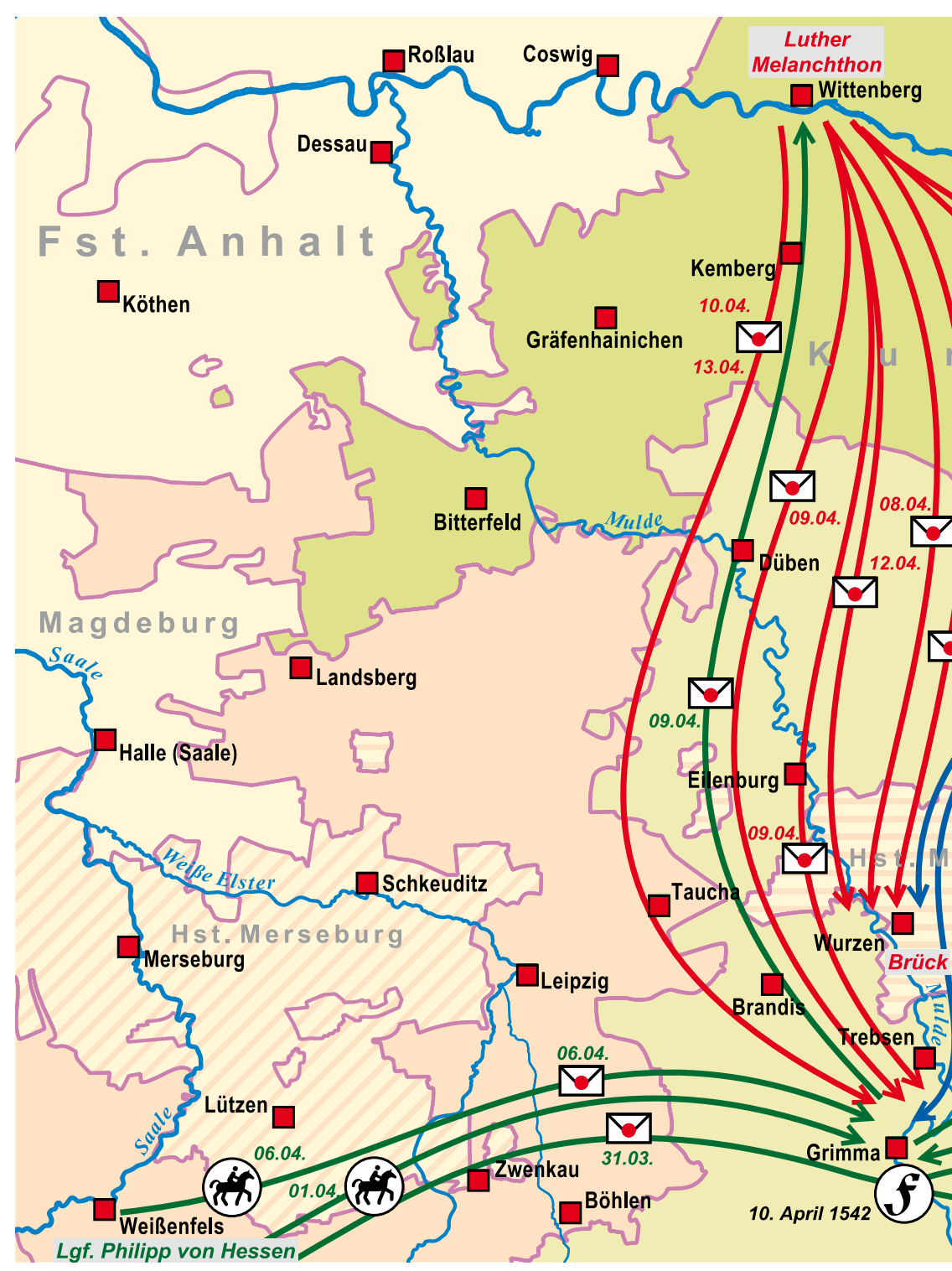

$$
\text { 30. März }
$$

$$
\text { 31. März }
$$

KF. JOHANN FRIEDRICH (Torgau) an Hz. Moritz (Dresden) Rechtfertigung des Vorgehens, Zurückweisung von Einmischung; Vorschlag zu rechtlicher Einigung
LGF. Philipp v. Hessen (Spangenberg) an Hz. Moritz (Dresden) Bitte um Stillhalten, Absicht gegen Besetzung von Wurzen, Bitte um Rat

BISCHOF JOHANN VON Meissen (Stolpen) an $\mathrm{Hz}$. Moritz (Dresden) Bitte um Hilfe gegen Kf. Johann von Sachsen 


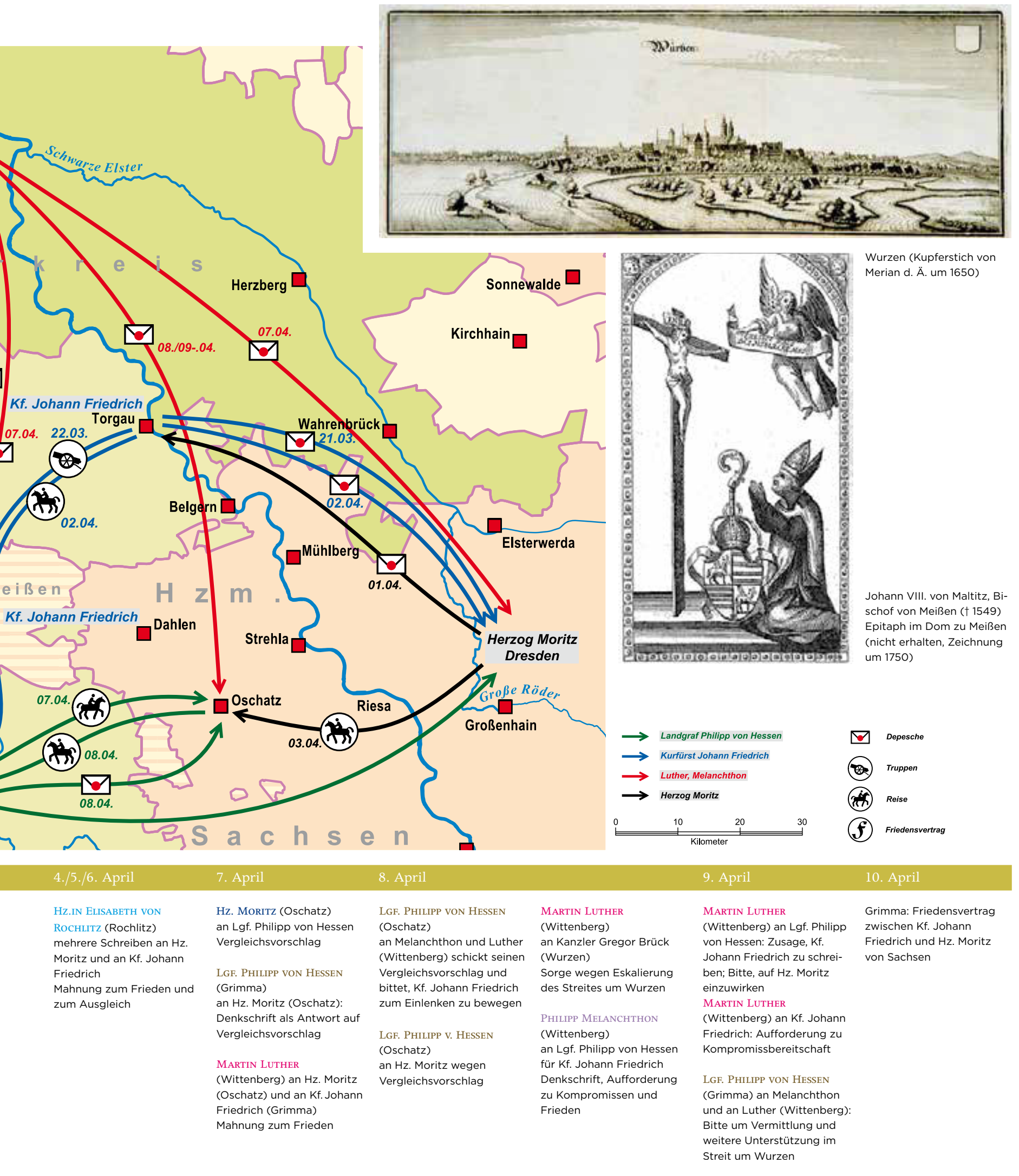

